Hope College

Hope College Digital Commons

Faculty Publications

2-27-2019

\title{
Bio-inspired Sensing and Actuating Architectures for Feedback Control of Civil Structures
}

Courtney A. Peckens

Hope College, peckens@hope.edu

I. Cook

C. Fogg

Follow this and additional works at: https://digitalcommons.hope.edu/faculty_publications

Part of the Biomedical Engineering and Bioengineering Commons

\section{Recommended Citation}

Repository citation: Peckens, Courtney A.; Cook, l.; and Fogg, C., "Bio-inspired Sensing and Actuating Architectures for Feedback Control of Civil Structures" (2019). Faculty Publications. Paper 1474.

https://digitalcommons.hope.edu/faculty_publications/1474

Published in: Bioinspiration \& Biomimetics, Volume 14, Issue 3, February 27, 2019. Copyright @ 2019 IOP Publishing, Bristol, United Kingdom.

This Article is brought to you for free and open access by Hope College Digital Commons. It has been accepted for inclusion in Faculty Publications by an authorized administrator of Hope College Digital Commons. For more information, please contact digitalcommons@hope.edu. 
ACCEPTED MANUSCRIPT

\section{Bio-inspired sensing and actuating architectures for feedback control of civil structures}

To cite this article before publication: Courtney Peckens et al 2019 Bioinspir. Biomim. in press https://doi.org/10.1088/1748-3190/ab033b

\section{Manuscript version: Accepted Manuscript}

Accepted Manuscript is "the version of the article accepted for publication including all changes made as a result of the peer review process, and which may also include the addition to the article by IOP Publishing of a header, an article ID, a cover sheet and/or an 'Accepted

Manuscript' watermark, but excluding any other editing, typesetting or other changes made by IOP Publishing and/or its licensors"

This Accepted Manuscript is @ 2018 IOP Publishing Ltd.

During the embargo period (the 12 month period from the publication of the Version of Record of this article), the Accepted Manuscript is fully protected by copyright and cannot be reused or reposted elsewhere.

As the Version of Record of this article is going to be / has been published on a subscription basis, this Accepted Manuscript is available for reuse under a CC BY-NC-ND 3.0 licence after the 12 month embargo period.

After the embargo period, everyone is permitted to use copy and redistribute this article for non-commercial purposes only, provided that they adhere to all the terms of the licence https://creativecommons.org/licences/by-nc-nd/3.0

Although reasonable endeavours have been taken to obtain all necessary permissions from third parties to include their copyrighted content within this article, their full citation and copyright line may not be present in this Accepted Manuscript version. Before using any content from this article, please refer to the Version of Record on IOPscience once published for full citation and copyright details, as permissions will likely be required. All third party content is fully copyright protected, unless specifically stated otherwise in the figure caption in the Version of Record.

View the article online for updates and enhancements. 


\title{
Bio-inspired sensing and actuating architectures for feedback control of civil structures
}

\author{
C A Peckens ${ }^{1}$, I Cook ${ }^{1}$, C Fogg $^{1}$ \\ ${ }^{1}$ Department of Engineering \\ Hope College, Holland, Michigan, USA \\ Email: peckens@hope.edu
}

\begin{abstract}
Civil structures, such as buildings and bridges, are constantly at risk of failure due to external environmental loads, such as earthquakes or strong winds. To minimize the effects of these loads, active feedback control systems have been proposed but such systems still face numerous challenges which impede their widespread adoption. In order to overcome many of these challenges, inspiration can be drawn from the signal processing and actuating techniques employed by the biological central nervous system to develop a bio-inspired control algorithm. In this study the front-end, signal processing techniques employed by biological sensory systems, and in particular the mammalian auditory system, are drawn upon in order to alleviate computations at the actuation node. This results in a simplistic control law that is a weighted combination of input information about the structure's response such that $\boldsymbol{F}=\boldsymbol{W N}$, where $\boldsymbol{F}$ is the applied control force, $\boldsymbol{W}$ is a pre-determined weighting matrix, and $\boldsymbol{N}$ is a deconstructed representation of the structural response to the applied excitation. There is no empirical solution for deriving an optimal weighting matrix, $\boldsymbol{W}$, and in this study numerous methods are explored in order to determine values for this matrix that produce the most effective control. These methods include particle swarm optimization, artificial neural networks, and optimal control theory. The various weighting matrices are integrated into the proposed bio-inspired control algorithm and applied in simulation to a five story benchmark structure. These methods are also compared to a traditional linear quadratic regulator (LQR) to gain insight into the overall performance of the bio-inspired control algorithm. Of the three training techniques, the particle swarm optimization technique offers the most effective control which is comparable in performance to the traditional LQR.
\end{abstract}

\section{Introduction}

Structural control is an attractive method for mitigating the undesired response of civil infrastructure (e.g., bridges, buildings) when subject to extreme load events, such as high winds or earthquakes. Rather than relying on dissipating energy through damage to the structure, feedback control systems aim to reduce structural response by applying counteracting forces using an actuating device [1]. Numerous researchers have demonstrated the effectiveness of such systems on civil structures using active control techniques [2]-[4]. Traditionally these systems have relied on numerous sensors distributed throughout the structure to transmit data via cables to a single controller that commands an actuator. As a result, all information has to be aggregated at a central location prior to the execution of the control law which can result in delays that inevitably degrade the control effectiveness. In an effort to overcome this, the architectural design of these systems has been extended to include multiple controllers and numerous semi-active actuators [3], [5], [6], but real-time communication between the sensors, controllers, and actuators is often still unachievable due to system constraints.

As such, researchers have explored replacing cables with wireless telemetry in control applications, partially due to the success of wireless sensor networks (WSNs) in monitoring many complex and largescale engineered systems such as bridges [7]-[10], buildings [11]-[13], wind turbines [14], [15], and 
ships [16], [17]. Due to their on-board microcontroller, wireless sensing units are capable of localized data processing, which allows them to serve as the controller in the actuation network. Additionally, they seamlessly interface with transducers, thus allowing them to serve as a sensing node, and actuators, which allows them to act as an actuating node, thereby increasing the overall flexibility of the network. Wireless telemetry has been successfully integrated into centralized control architectures [18]-[21], but these studies also highlight the challenges of the technology, such as a higher probability of data loss during transmission. Similar to the information bottleneck experienced with cabled systems, this data loss can degrade real-time execution of control algorithms, thus impeding the overall effectiveness [22].

In an attempt to address these limitations, researchers have explored pairing WSNs with decentralized control architectures and localized actuation. In decentralized control, a large-scale control system is divided into small sub-systems that use local and neighboring sensor data to make control decisions. Because WSNs are capable of peer-to-peer communication, they are well suited for decentralized control as this eliminates the need for a centralized controller and creates countless possible control architectures. In [23], a partially decentralized Linear Quadratic Regulator (LQR) control scheme that leverages a Kalman filter to estimate unknown system states was validated on a full scale structure. In [24], a sparse representation of the LQR was proposed which requires less information for decision-making than a traditional centralized approach, thus reducing information flow requirements. In [25], the authors proposed a distributed $H_{\infty}$ algorithm for civil infrastructure and in [26], the authors explored distributing the $H_{\infty}$ algorithm across multiple communication subnets of wireless sensing nodes. While these studies have demonstrated the successful use of WSNs in decentralized control architectures they also highlight several challenges associated with this technology, such as increased computational requirements at the already resource-constrained sensing node or decision-making based on reduced information, resulting in some degradation in control effectiveness. Furthermore, while wireless structural control has been validated on a variety of experimental test beds, all of these/applications have been performed within the confines of laboratories and few have been extended to real-world structures and long-term deployments. As such, in order to effectively implement wireless structural control it is imperative that the current challenges of the technology be addressed. One potential strategy for overcoming current deficiencies is to draw inspiration from the method by which biological systems sense and actuate, thus resulting in a novel bio-inspired sensing and actuating paradigm.

Biological systems are capable of integrating sensing with actuation in a simplistic manner that is desirable for engineered sensing and actuating systems. Within biological systems, information is received from external stimulus through multiple receptors [27]. Receptor neurons transmit this information to layers of processing neurons where the data is further integrated and manipulated through basic operations, such as addition, subtraction, multiplication, or filtering [28]. Finally motor neurons receive this information from a layer of processing neurons and activate their associated muscles, thus initiating actuation in the system [29]. In this paper, inspiration is drawn from the processes utilized by biological sensory and actuation systems to overcome the limitations found in equivalent engineering systems. In particular, a bio-inspired control algorithm is proposed that uses the front-end signal processing employed by the auditory system to streamline computations at the actuation node.

\section{Actuation in Biological Systems}

Sensing and actuation in biological systems is a streamlined and efficient process which starts when information is perceived by various sensory receptors. Sensory receptors are tuned to specific input stimuli (e.g., frequency, pressure, light) [27]. While each class of sensory receptors forms networks with subsequent neurons in distinct structures in the central nervous system, in general, connections between layers of neurons follow similar patterns (Figure 1a). The receptor neuron transmits information about the stimulus to a sensory neuron via a graded potential (Figure 1b), which is an electrical signal with constant amplitude whose duration encodes the amplitude of the input signal [30]. The sensory neuron converts the received information to a series of action potentials (Figure 1c), or electrical pulses of 


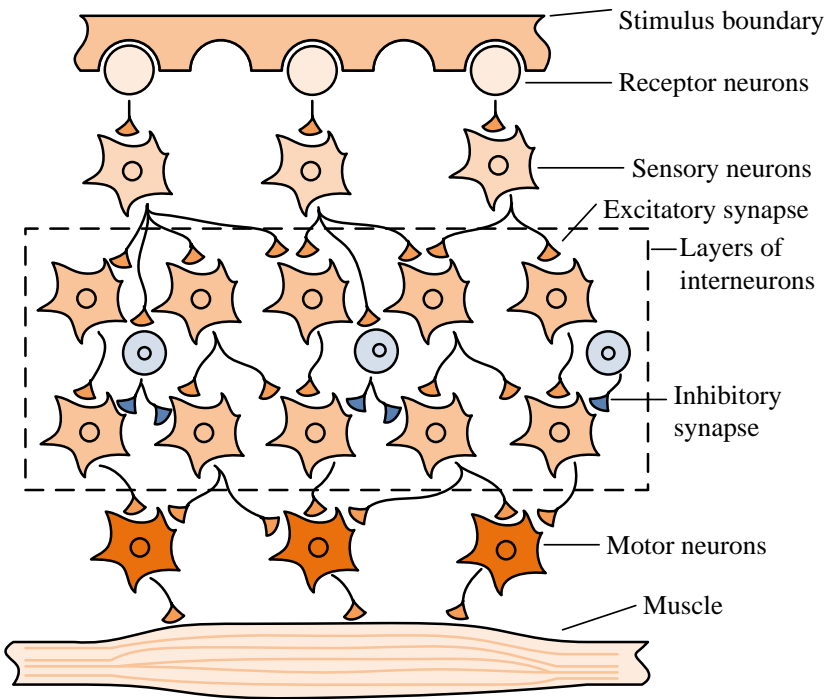

(a)

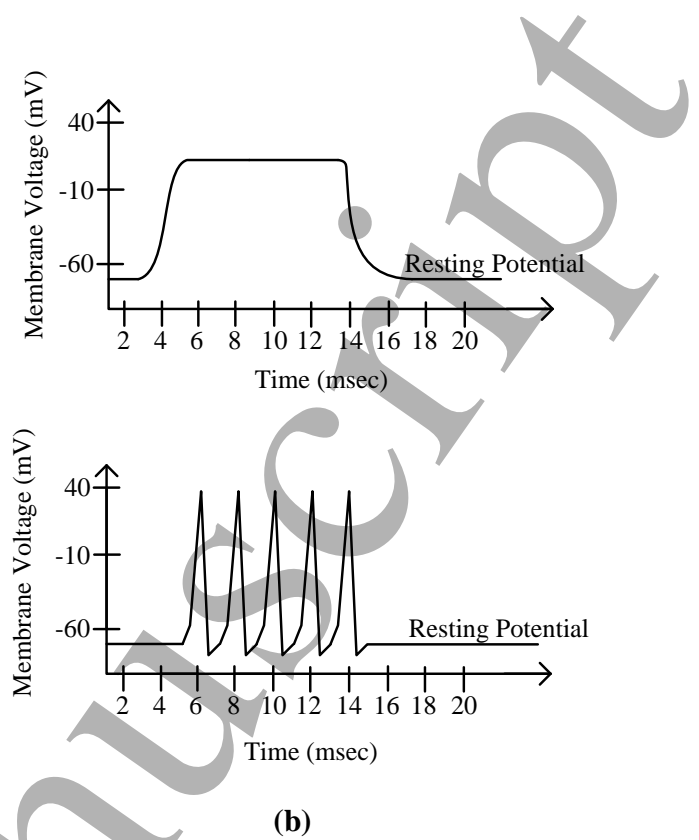

(b)

Figure 1. Generalized structure of neurons used for sensing and actuation in biological sensory system (a), where receptors communicate the perceived stimulus to the sensory neurons via graded potentials ( $b$, top) and all other communication occurs between neurons via action potentials (b, bottom).

constant amplitude, and transmits these to subsequent layers of neurons, termed interneurons. The frequency and overall duration of the action potentials encode the amplitude of the received signal [31]. The interneurons aggregate information from multiple sources based on the connection type (i.e., excitatory or inhibitory) between the transmitting and receiving neurons. Excitatory connections further promote decisions while inhibitory connections work to inhibit decisions and help to provide spatial detail in sensory systems [32]. Depending on the complexity of the required decision making, this process may continue through several layers of neurons, whereby each layer further integrates information through simple connections, until reaching the level of the motor neuron. For basic organisms with a minimal number of neurons, such as the leech or C. Elegan, the hierarchy of neurons and connection types can be mapped out, thus demonstrating the complete sensing and actuation process of these life forms [33], [34]. As organisms become more advanced, however, this mapping becomes increasingly complex.

To complete the sensing and actuation process in biological systems, motor neurons convey information to muscle fibers which are typically responsible for actuation in biological systems [29]. Similar to the connection between two neurons, the connection between a motor neuron and the muscle is a chemical synapse. Information is transmitted from the motor neuron through its axon (Figure 2) via action potentials. These action potentials induce neurotransmitters to flow from the presynaptic terminal on the motor neuron and into the postsynaptic membrane on the muscle. When these transmitters cross the synaptic cleft from the motor neuron to the muscle an action potential is initiated that travels the length of the muscle. Muscles are comprised of bundles of muscle fibers and a single motor neuron can activate hundreds of these fibers at one time. The connection type (i.e., excitatory or inhibitory) between the motor neuron and each muscle fiber plays an important role in the overall response of the system. For example, it has been found that if the leech senses an external pressure on one side of its body, the motor neurons closest to the pressure are excited, thus causing contraction, while the motor neurons on the opposite side of its body are inhibited, thus causing relaxation [35]. By having both excitatory and inhibitory motor neuron connections, the leech is able to more effectively bend away from the stimulus. 


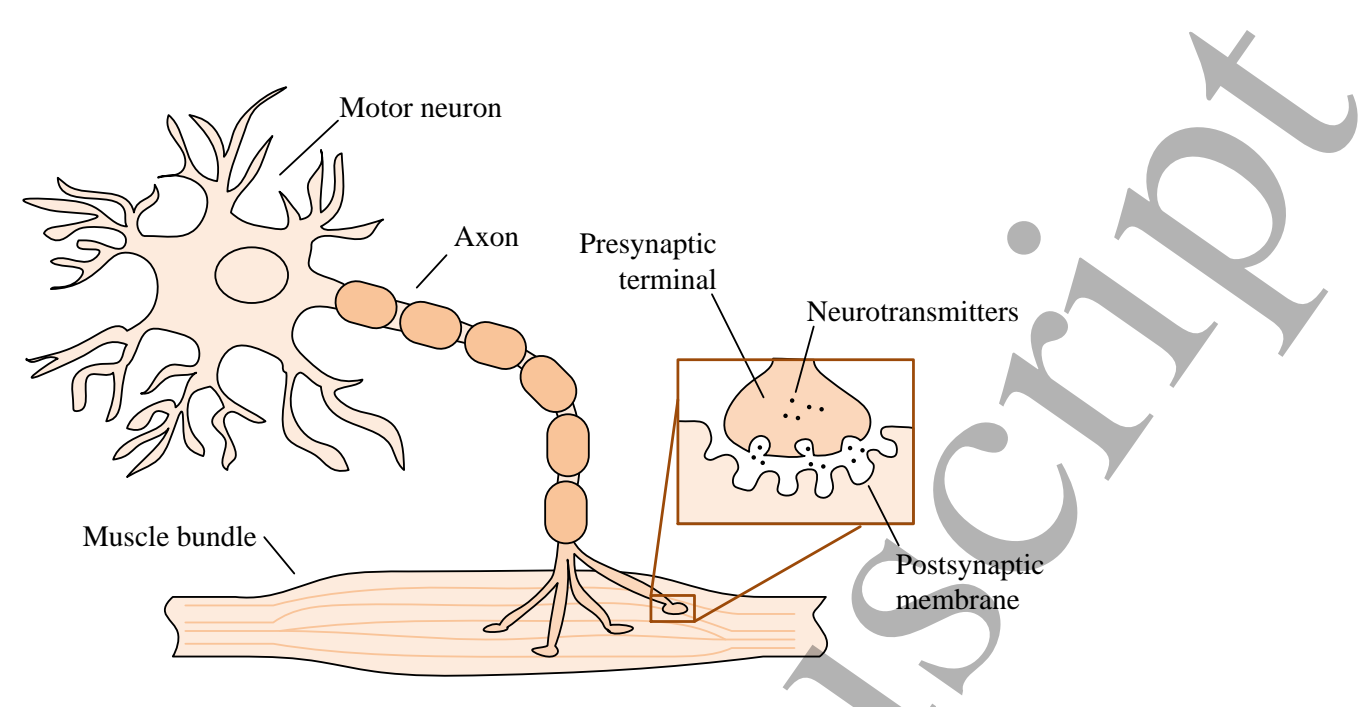

Figure 2. Connection between motor neuron and muscle

A motor neuron dictates the magnitude of the force to be exerted by using both rate coding and the size principle. First, the motor neuron uses rate coding by sending a series of action potentials with each successive spike increasing the intensity of the muscle actuation, up to a limit [36]. Second, to increase the overall intensity of the muscle actuation, motor neurons are recruited in an orderly manner by the motor cortex depending on their physiological properties [37], [38]. As such, the overall intensity of the actuation increases as the number of activated motor neurons increases. Once commands have been sent from motor neurons to the muscle fibers, the neuron uses feedback mechanisms to ensure that the overall desired response is achieved and to fine-tune the actuation based on this information [39]. This enables effective actuation for a variety of input stimulus. Therefore, all muscle actuation depends on both the commands from motor neurons as well as the connection type between the neuron and the fiber. In this paper, the simplistic basis by which organisms respond to external stimuli through muscle actuation will be mimicked. In particular, the inhibitory and excitatory connections of these systems will be adapted to establish synaptic strength connections between sensor nodes and motor neuron nodes. These synaptic values will vary in magnitude which is similar to muscle recruitment of multiple muscle fibers. Multiple techniques for developing these synaptic strength values will be explored and their effectiveness will be evaluated in simulation.

\section{Bio-Inspired Control Architecture}

Actuation in biological systems relies on data transmission and information integration that starts with the sensory neurons, works through the interneuron layer, and ends with the motor neurons (Figure 1a). The receptor neurons initiate the process and in the bio-inspired engineered system a novel sensing node inspired by the mammalian auditory system will be used as the input layer. This sensor, first proposed by Peckens et al. [40], draws inspiration from the signal processing techniques employed by the cochlea within the auditory system to enable real-time frequency decomposition of convoluted signals [41]. As discussed by Peckens and Lynch [42], each cochlea-inspired sensing node contains multiple "neurons" that process and transmit data simultaneously. By enabling information extraction at the receptor nodes through real-time pre-processing, the actual control law at the motor neuron node is streamlined, thus alleviating the computational requirements at the node and overcoming one of the current challenges that plagues control systems in civil infrastructure.

The founding principle of this streamlined architecture is the information integration between layers of neurons using synaptic strengths (i.e., amplification or attenuation factors), as well as the aggregation of information from multiple sources [43], [44]. This structure is mimicked in the bio-inspired control law 
Figure 3. Bio-inspired sensing and actuating architecture

with motor neuron nodes aggregating information from multiple receptor nodes using weighting values which can be both positive, for amplification, and negative, for attenuation (Figure 3). The cochleainspired sensing node is utilized at the receptor layer which enables real-time spectral decomposition of the input information. Due to this front-end processing at the receptor node no further signal processing is required at the motor neuron node and instead it can apply a control force, $F_{k}$, that is simply a weighted aggregation of the received information,

$$
F_{k}=\sum_{i=1}^{n} \sum_{j=1}^{b} W_{i j k} N_{i j}
$$

where $W_{i j k}$ is the synaptic strength between the $j^{\text {th }}$ neural unit for $b$ units on the $i^{\text {th }}$ receptor node for $n$ nodes and the $k^{\text {th }}$ motor neuron node for $p$ nodes. $N_{i j}$ is the output from the $j^{\text {th }}$ neural unit on the $i^{\text {th }}$ receptor node. In this architecture $p$ represents the number of actuating nodes in the system, while $n$ represents the number of sensing nodes. It is possible for $p$ to equal $n$ but it is not necessary. Developing the weighting matrix, $\boldsymbol{W}$, is integral to the success of the control law and three different techniques for optimizing these values are evaluated in simulation in this paper: bio-inspired optimal control theory (BIO-LQR), artificial neural networks (ANNs), and particle swarm optimization (PSO).

\subsection{State Space System Model}

Prior to exploring these methods, however, the system model must be defined. The base-excited structural system is modeled in continuous time as an $n$ degree-of-freedom, linear time-invariant, lumped mass shear structure. The can be generalized through $n$ equations of motion

$$
\boldsymbol{M} \ddot{\boldsymbol{x}}(t)+\boldsymbol{C}_{\boldsymbol{d}} \dot{\boldsymbol{x}}(t)+\boldsymbol{K}_{s} \boldsymbol{x}(t)=-\boldsymbol{M i} \ddot{x}_{g}(t)+\boldsymbol{L F}(t)
$$

where $\boldsymbol{M}, \boldsymbol{C}_{\boldsymbol{d}}$, and $\boldsymbol{K}_{\boldsymbol{s}} \in \mathbb{R}^{n \times n}$ are the mass, damping and stiffness matrices, respectively. The displacement vector relative to the base of the structure is $\boldsymbol{x} \in \mathbb{R}^{n}, x_{g}$ is the ground displacement, and $\imath \in \mathbb{R}^{n}$ is the ground acceleration influence vector, where each term is unitary. The locations of the actuators are described by the matrix, $\boldsymbol{L} \in \mathbb{R}^{n \times p}$, and $\boldsymbol{F} \in \mathbb{R}^{p}$ is a vector of control forces, where $p$ is the number of input control forces. The variable $t$ represents continuous time.

The equation of dynamic equilibrium described in Equation 2 can be represented in state space form as

$$
\dot{\mathbf{z}}=A \boldsymbol{z}+\boldsymbol{B u}+G \ddot{x}_{g}
$$




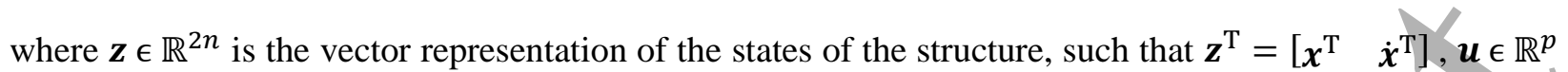
is the vector of input control forces (equivalent to $\boldsymbol{F}$ in Equation 1). $\boldsymbol{A} \in \mathbb{R}^{2 n \times 2 n}$ is the state transition matrix, defined as

$$
\boldsymbol{A}=\left[\begin{array}{cc}
\mathbf{0} & \boldsymbol{I} \\
-M^{-1} \boldsymbol{K}_{s} & -M^{-1} C_{d}
\end{array}\right],
$$

where $I$ is the identity matrix. $\boldsymbol{B} \in \mathbb{R}^{2 n \times p}$ is the control matrix and $\boldsymbol{G} \in \mathbb{R}^{2 n}$ is the ground input matrix, where

$$
B=\left[\begin{array}{c}
0 \\
M^{-1} L
\end{array}\right], G=\left[\begin{array}{c}
0 \\
-l
\end{array}\right]
$$

The output vector, $\boldsymbol{y} \in \mathbb{R}^{q}$, is based on the sensors that are available to measure the response of the structure as they relate to the states, $z$, such that

$$
\boldsymbol{y}=\boldsymbol{C z}+\mathrm{Du}+\boldsymbol{H} \ddot{x}_{g}
$$

given that $\boldsymbol{C} \in \mathbb{R}^{q \times 2 n}$ is the measurement output matrix, $\boldsymbol{D} \in \mathbb{R}^{q \times p}$ is the control feedforward matrix, and $\boldsymbol{H} \in \mathbb{R}^{q}$ is the ground feedforward matrices.

\subsection{Integration with the cochlea-inspired sensing node}

To fully exploit the streamlined bio-inspired control architecture, the cochlea-inspired sensing node is used as the receptor node, thus leveraging its real-time frequency decomposition and parallel processing capabilities. The cochlea-inspired sensing node consists of "neurons" that simultaneously bandpass the input signal through overlapping passbands. The sinusoidal output of each filter is tracked by a unique computing core which implements a real-time peak-picking algorithm. When a peak is detected, it is immediately broadcasted to the motor neuron node. Each peak is considered to be the biological equivalent to a spike train signal used by neurons. Once received at the motor neuron node, the detected peak values are weighted according to their source and then summed, resulting in an actuating force, $\boldsymbol{F}$. This complete process is summarized in Figure 4; further details can be found in [40], [42]. Due to the on-board processing that occurs on the cochlea-inspired sensing node, the motor neuron node never

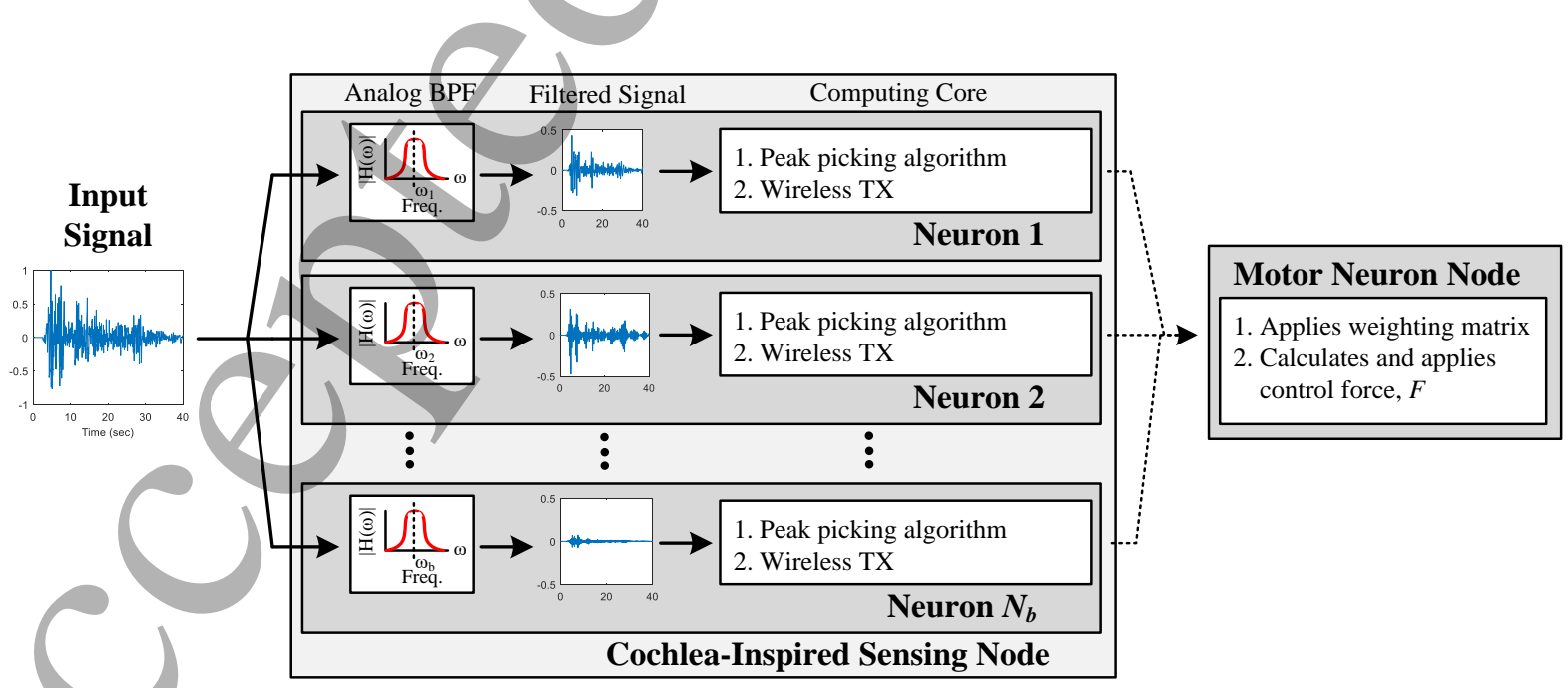

Figure 4. Functional schematic of single cochlea-inspired sensing node integrated with motor neuron node; all neuron boards are simultaneously presented with the input signal, resulting in parallel processing at the node. BPF $=$ bandpass filter. 
receives a traditional Nyquist-sampled representation of the input signal or even the filtered signals after passing through the filter bank. Instead, the motor neuron node only receives the peak values of the filtered signals and these are directly weighted using Equation 1. While it is anticipated that this will result in a slight degradation of the overall control performance of the system, the computational requirements at the motor neuron node are greatly reduced, thus minimizing time delays and further promoting real-time control.

It is assumed that the inter-story drift of each floor of the structure is measured using a cochlea-inspired sensing node. To optimize the functionality of the cochlea-inspired sensing node, the bandwidth and filter spacing of each bandpass filter on the "neuron" can be modified to best capture the dynamics of the given system. Based on a parametric optimization previously conducted by Peckens et al. [40], these were chosen to be $0.5 \mathrm{~Hz}$ and $0.7 \mathrm{~Hz}$, respectively. Similarly, the number of filters in each node can be modified so as to fully capture the dynamic range of the structure as dictated by its modal response.

The on-board filtering of the "neurons" introduces additional dynamics into the model which must be included. In order to properly model these dynamics, the state space equations (Equations 3 and 4) are extended to include the cochlea-inspired sensing nodes, which is a function of the bandpass filtering effect described through the transfer function,

$$
\mathcal{F}_{i}(s)=\frac{2 \xi_{i} \omega_{i} s}{s^{2}+2 \xi_{i} \omega_{i} s+\omega_{i}^{2}}
$$

where $\xi_{i}$ is the damping ratio and $\omega_{i}$ is the center frequency of the $i^{\text {th }}$ filter. In this case, the input to the transfer function is the inter-story displacement of a given floor and the output is the filtered component of the input signal at the given filter center frequency. The dynamics of the entire system including the structure and the sensing nodes can be represented by expanding the original state space equations to now include the states of the $n$ cochlea-inspired sensing nodes,

$$
\begin{array}{r}
\dot{z z}=\widehat{A} z z+\widehat{B} u+\widehat{G} \ddot{x}_{g} \\
y y=\widehat{C} z+\widehat{D} u+\widehat{H} \ddot{x}_{g}
\end{array}
$$

where $\boldsymbol{z z}^{\mathrm{T}}=\left[\begin{array}{llll}\boldsymbol{x}^{\mathrm{T}} & \boldsymbol{x}_{\boldsymbol{c s}}^{\mathrm{T}} & \dot{\boldsymbol{x}}^{\mathrm{T}} & \dot{\boldsymbol{x}}_{\boldsymbol{c s}}^{\mathrm{T}}\end{array}\right]$ such that $x_{c s, i}$ is the state representing the output of the $i^{\text {th }}$ filter board for $i=1, \ldots, b \times n$, which represents the total number of filter boards across all sensor nodes, and $\boldsymbol{y} \boldsymbol{y} \in \mathbb{R}^{b n}$ is a vector containing the output of each filter board on each cochlea-inspired sensing node. With the addition of the cochlea-inspired sensing nodes, the system matrix is expanded to become

$$
\widehat{A}=\left[\begin{array}{cccc}
0 & 0 & \mathrm{I} & 0 \\
\mathbf{0} & \mathbf{0} & \mathbf{0} & \mathrm{I} \\
-\boldsymbol{M}^{1} K_{\mathcal{S}} & \mathbf{0} & -\boldsymbol{M}^{1} \boldsymbol{C}_{\boldsymbol{d}} & \mathbf{0} \\
\mathbf{0} & -\boldsymbol{X} & -\boldsymbol{\Psi} & -\boldsymbol{r}
\end{array}\right] \in \mathbb{R}^{2(n+b n) \times 2(n+b n)}
$$

The matrices $\boldsymbol{X} \in \mathbb{R}^{b n \times b n}, \boldsymbol{\Psi} \in \mathbb{R}^{b n \times n}$, and $\boldsymbol{\Upsilon} \in \mathbb{R}^{b n \times b n}$ are populated based on each filter's dynamics, as described by Equation 5. In particular,

$$
X=\left[\begin{array}{cccc}
\chi_{1} & \mathbf{0} & \cdots & \mathbf{0} \\
\mathbf{0} & \chi_{2} & \cdots & \mathbf{0} \\
\vdots & \vdots & \ddots & \mathbf{0} \\
\mathbf{0} & \mathbf{0} & \mathbf{0} & \chi_{n}
\end{array}\right], \Psi=\left[\begin{array}{cccc}
\psi_{1} & \mathbf{0} & \cdots & \mathbf{0} \\
\mathbf{0} & \boldsymbol{\psi}_{2} & \cdots & \mathbf{0} \\
\vdots & \vdots & \ddots & \mathbf{0} \\
\mathbf{0} & \mathbf{0} & \mathbf{0} & \boldsymbol{\psi}_{n}
\end{array}\right] \text {, and } \boldsymbol{Y}=\left[\begin{array}{cccc}
v_{1} & \mathbf{0} & \cdots & \mathbf{0} \\
\mathbf{0} & \boldsymbol{v}_{2} & \cdots & \mathbf{0} \\
\vdots & \vdots & \ddots & \mathbf{0} \\
\mathbf{0} & \mathbf{0} & \mathbf{0} & \boldsymbol{v}_{n}
\end{array}\right]
$$


where $\chi_{i}=\operatorname{diag}\left\{\omega_{i 1}{ }^{2} \quad \omega_{i 2}{ }^{2} \quad \cdots \quad \omega_{i b}{ }^{2}\right\} \in \mathbb{R}^{b \times b}, \boldsymbol{\psi}_{i}=\left[\begin{array}{llll}2 \xi_{i 1} \omega_{i 1} & 2 \xi_{i 2} \omega_{i 2} & \cdots & 2 \xi_{i b} \omega_{i b}\end{array}\right]^{\mathrm{T}} \in \mathbb{R}^{b}$, and $\boldsymbol{v}_{i}=\operatorname{diag}\left\{2 \xi_{i 1} \omega_{i 1} \quad 2 \xi_{i 2} \omega_{i 2} \quad \cdots \quad 2 \xi_{i b} \omega_{i b}\right\} \in \mathbb{R}^{b \mathrm{xb}}$, given that $\operatorname{diag}\{\cdot\}$ represents a diagonal matrix.

In equation 7 , the output matrix, $\widehat{\boldsymbol{C}}$, is manipulated to make the states associated with the filter output, or $\boldsymbol{x}_{\boldsymbol{c s}}$, the output variables. The cochlea-inspired sensing nodes do not affect $\boldsymbol{B}, \boldsymbol{D}, \boldsymbol{G}$, or $\boldsymbol{H}$, other than expanding them to account for additional states; as such, zeros are added that correspond to the states $\boldsymbol{x}_{\boldsymbol{c s}}$ and $\dot{\boldsymbol{x}}_{\boldsymbol{c s}}$ to form $\widehat{\boldsymbol{B}}, \widehat{\boldsymbol{D}}, \widehat{\boldsymbol{G}}$, and $\widehat{\boldsymbol{H}}$.

\subsection{Bio-Inspired Control Weighting Matrix}

The applied actuating force, $\boldsymbol{F}(t)$, is formulated as a weighted sum of the outputs of the cochlea-inspired sensing nodes. Three techniques are employed to determine appropriate weighting values, including optimal control theory, artificial neural networks, and particle swarm optimization.

\subsubsection{Optimal Control Theory}

In the first approach, the synaptic weights are developed using well-established optimal control theory by leveraging the Linear Quadratic Regulator (LQR) [45] to establish scaling factors between the input sensing data, or receptor nodes, and the actuating output, or motor neuron node. LQR uses the algebraic Riccati equation to minimize the cost function

$$
\mathrm{J}=\int_{0}^{\infty}\left(z^{\mathrm{T}} \boldsymbol{Q}+\boldsymbol{u}^{\mathrm{T}} \boldsymbol{R} \boldsymbol{u}\right) d t
$$

subject to the full state feedback control law, $\boldsymbol{u}=-\boldsymbol{K} \boldsymbol{z}$, where $\boldsymbol{K} \in \mathbb{R}^{p \times m}$ is the resulting constant feedback gain matrix, given $m$ states and $p$ control forces. This minimization is subject to two parameters: $\boldsymbol{Q} \in \mathbb{R}^{m \times m}$ which applies a weight to the cost of the structural response and $\boldsymbol{R} \in \mathbb{R}^{p \times p}$ which applies a weight to the cost of control effort.

When considering control of civil infrastructure, however, it is often difficult to measure all of the states in the system (i.e., displacement and velocity) without implementing computationally expensive observers such as the Kalman filter [46] and thereby, reducing the real-time capabilities of the system. As such, the traditional LQR is adapted for optimal control using output-state feedback, $\boldsymbol{u}=\boldsymbol{K} \boldsymbol{y}$, such that $\boldsymbol{K} \in \mathbb{R}^{p \times q}$ where $q$ is the number of output states. For the bio-inspired control law these outputs states correspond to the output of the "neurons" on the cochlea-inspired sensing node such that $q$ is equivalent to $n \times b$. The modifications to the performance index, as well as analysis for solving for the $\boldsymbol{K}$ matrix can be found in more detail in [47]. The resulting $\boldsymbol{K}$ matrix for output-state feedback represents the weighting matrix in the bio-inspired control algorithm, or $\boldsymbol{W}$ in Equation 1.

\subsubsection{Artificial Neural Networks}

Researchers have long been interested in the methods by which biology performs streamline signal processing through complex networks of interconnected computing nodes and this has been encapsulated in the well-established field of artificial neural networks (ANNs). A standard ANN consists of many simple, connected processors called neurons which produce real-value activation outputs. Typically networks of these processors are constructed to include an input layer, at least one hidden layer, and then an output layer. Each subsequent layer of neurons is activated through weighted connections from the previous layer. If designed properly, ANNs can be used to efficiently model the nonlinear behavior of virtually any system [48], [49], including a wide range of engineering applications across numerous engineering disciplines.

For the purposes of this study, a feedforward ANN is applied to the bio-inspired control theory in order to develop the weighting matrices between the receptor nodes and the motor neuron nodes. The structure of 
the ANN consists of one input layer, one hidden layer, and one output layer. The output of each hidden node in the network is defined mathematically as

$$
y_{H, j}=f_{j}\left(\sum w_{j i} y_{I, i}+b_{j}\right)
$$

where $y_{I, i}$ is the input data from the $i^{\text {th }}$ node in the input layer, $y_{H, j}$ is the output data from the $j^{\text {th }}$ node in the hidden layer, $w_{j i}$ is the weight between the $i^{\text {th }}$ input layer node and the $j^{\text {th }}$ hidden layer node, $b_{j}$ is the bias value at the $j^{\text {th }}$ node, and $f_{j}(x)$ is its activation function [50]. A similar relationship is created between the hidden layer and the output layer,

$$
y_{O, k}=f_{k}\left(\sum w_{k j} y_{H, j}+b_{k}\right)
$$

where $y_{O, k}$ is the output data from the $k^{\text {th }}$ node in the output layer and equivalent to $\boldsymbol{F}$ in Equation 1.

The sigmoid function,

$$
f_{j}(x)=\frac{1}{1+e^{-x}}
$$

is used as the activation function in the hidden layer, as it introduces a non-linearity in the estimated output and is commonly used for regression applications. A linear function, $f_{k}(x)=x$, is chosen as the activation function for the output layer and therefore does not introduce any additional nonlinearities into the system.

The weighting values between layers of neurons are established using stochastic gradient descent with traditional backpropagation training methods [50]. To achieve this, the gradient of an error function, $E_{k}$, is used to alter the weighting and bias values of the neural network in the direction of the negative cost gradient, where

$$
E_{k}=\frac{1}{2}\left(T_{k}-y_{O, k}\right)^{2}
$$

such that $T_{k}$ is the target output of the $k^{\text {th }}$ node. Numerous training cases are presented to the ANN, and the weights and bias vectors between the layers of nodes are iteratively updated. As the ANN contains a hidden layer of nodes, as well as two weighting matrices, bias vectors, and activation functions, this method does not follow the same architecture that is proposed in Figure 3. However, this algorithm still maintains the same function of Figure 3 in that peak values are transmitted from the cochlea-inspired sensing nodes and then transformed into an output control force after passing through this multi-layer network.

\subsubsection{Particle Swarm Optimization}

A third approach for optimizing the weighting matrix in the bio-inspired control algorithm focuses on utilizing particle swarm optimization (PSO), which is an iterative learning technique that draws inspiration from biology and is capable of optimizing continuous nonlinear functions. In PSO, a number of solutions, or particles, are dispersed randomly in a search space and each particle location is evaluated according to a specified objective function. Depending on its own history, as well as the behavior of other nearby particles, each particle moves to a new location in the search space with each iteration of the algorithm, with the goal of moving closer to the optimum of the objective function [51].

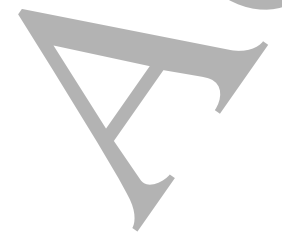


To achieve this, each particle in the swarm tracks three vectors: $\boldsymbol{x}$ which represents the current position, $\boldsymbol{v}$ which is the current velocity, and $\boldsymbol{x}_{\boldsymbol{b}}$ which is the previous best position. These three vectors are the dimension of the search space. Each particle also interacts with neighboring particles and stores the position that best optimizes the objective function, denoted as $\mathbf{g}$, in order to leverage the benefits of the swarm. Each particle updates its three vectors every iteration through the equations:

$$
\begin{gathered}
\boldsymbol{v}_{\boldsymbol{i}}(k+1)=\omega \boldsymbol{v}_{\boldsymbol{i}}(k)+\rho_{1} \gamma_{1}\left(\boldsymbol{x}_{\boldsymbol{b}, \boldsymbol{i}}(k)-\boldsymbol{x}_{\boldsymbol{i}}(k)\right)+\rho_{2} \gamma_{2}\left(\boldsymbol{g}(k)-\boldsymbol{x}_{\boldsymbol{i}}(k)\right) \\
\boldsymbol{x}_{\boldsymbol{i}}(k+1)=\boldsymbol{x}_{\boldsymbol{i}}(k)+\boldsymbol{v}_{\boldsymbol{i}}(k+1) \\
\lambda=\lambda \times \tau
\end{gathered}
$$

where $i$ is the particle number, $k$ is the iteration number, $\rho_{l}$ and $\rho_{2}$ are random numbers between 0 and 1 , and $\gamma_{1}$ and $\gamma_{2}$ are the acceleration coefficients which are both assigned to be 2 as recommended in [51]. Equation 13 also includes an inertia weight, $\lambda$, which affects the convergence and plays a role in balancing local versus global search of the particles [52], as well an inertia damping constant, $\tau$, which gradually modifies this balance. The inertia coefficient, $\lambda$, is initially assigned to be 1 , and is decreased using a damping constant, $\tau$, of 0.99 [53], resulting in a preliminary global search that gradually becomes more localized. In this study, each particle represents a potential weighting matrix (equivalent to $W$ in Equation 1) between the receptor nodes and the motor neuron nodes in the bio-inspired control theory. The particle values which produce the lowest global function output in the final iteration represent the synaptic strength values found within this weighting matrix.

\subsection{5-Story Benchmark}

The bio-inspired control theory is validated on a model of the 5-story Kajima-Shizuoka building (Figure 5, Table 1). The model is similar to the lumped mass system used by Wang [54], which is a modified version of the Kajima-Shizuoka building presented by Kurata et al. [6]. A classical damping matrix using Rayleigh damping that is both mass-proportional and stiffness-proportional is assumed, using 5\% natural damping [55]. It is also assumed that only horizontal degrees-of-freedom are measured and controlled, while vertical and rotational degrees of freedom are considered negligible. As such, the states of the system as defined in Equation 3 are horizontal displacements and velocities at each floor. Each floor is assumed to include an installed transducer that measures inter-story displacement, which is input into a cochlea-inspired sensing node, as well as an ideal actuator. It also assumed that these nodes communicate detected peak values to motor neuron nodes on all floors, which command their respective actuators.

The effectiveness of the bio-inspired control theory is evaluated using multiple methods for creating the synaptic weights, including BIO-LQR, ANN, and PSO, and these are compared against a more traditional controller that employs a full state LQR. Four cost functions, adapted from Ohtori et al. [56], are used to characterize the effectiveness of these different controllers in reducing the structure's seismic response with respect to inter-story drift and floor acceleration when normalized to the uncontrolled structural response due to the same seismic excitation. Minimization of inter-story drift is important as it reduces the likelihood of damage to the building system, especially to nonstructural elements such as partitions and windows. Floor acceleration is related to the force exerted on the structure, as well as occupational comfort during the event. The minimization of these two parameters is quantified according to two cost functions; one cost function compares absolute maximum values, while the other compares the vector norm of the response over the entire test period. The cost functions are given as

$$
\boldsymbol{J}_{\mathbf{1}}=\frac{\max \left(\left|\boldsymbol{d}(t)_{\text {controlled }}\right|\right)}{\max \left(\left|\boldsymbol{d}(t)_{(\text {uncontrolled }}\right|\right)}
$$




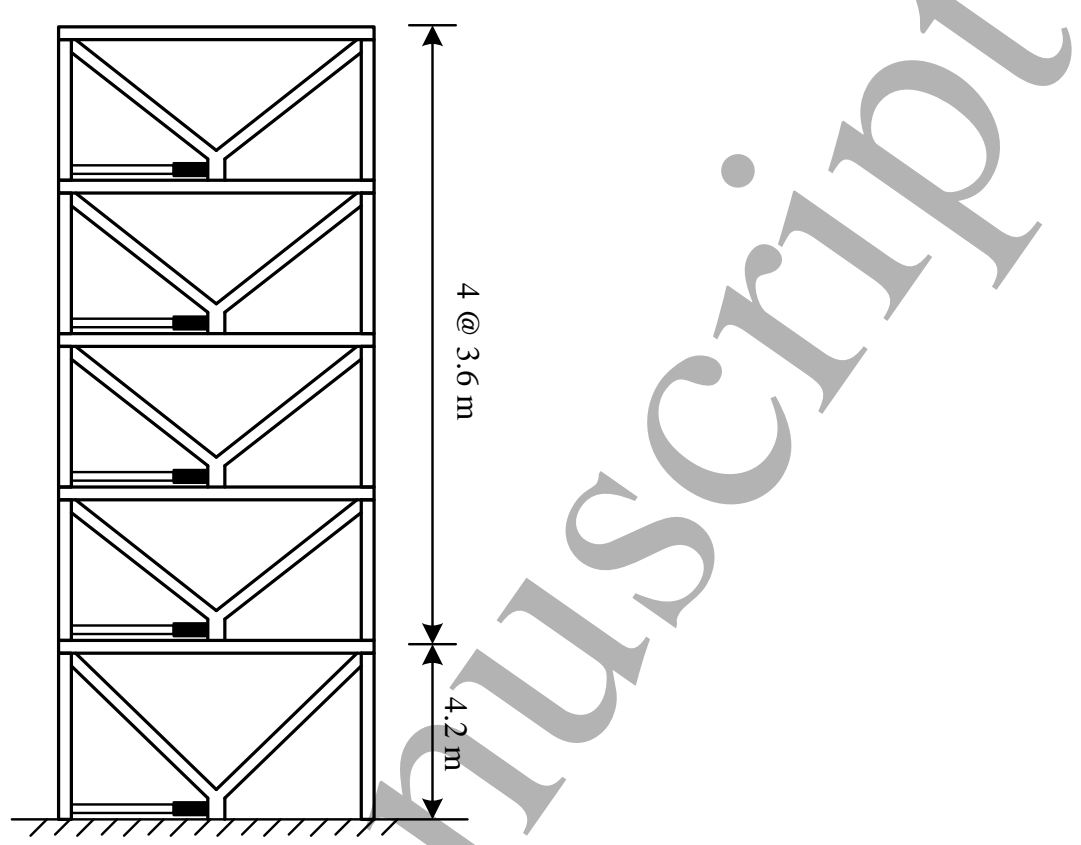

Figure 5. Five story benchmark structure [6], including one actuator at each story. The five natural frequencies are 1.00, $2.82,4.49,5.80$, and $6.77 \mathrm{~Hz}$.

Table 1. Five story benchmark structure properties; structural damping ratio is $5 \%$

\begin{tabular}{ccc} 
Floor & Seismic mass $(\mathrm{kg})$ & Interstory stiffness $(\mathrm{kN} / \mathrm{m})$ \\
\hline 1 & $215.2 \times 10^{3}$ & $147 \times 10^{3}$ \\
2 & $209.2 \times 10^{3}$ & $113 \times 10^{3}$ \\
3 & $207.0 \times 10^{3}$ & $99 \times 10^{3}$ \\
4 & $204.8 \times 10^{3}$ & $89 \times 10^{3}$ \\
5 & $266.1 \times 10^{3}$ & $84 \times 10^{3}$ \\
\hline
\end{tabular}

where $\boldsymbol{d}$ is the time history of the inter-story drift for all floors, and

$$
\boldsymbol{J}_{2}=\frac{\left\|\boldsymbol{d}(t)_{\text {controlled }}\right\|}{\left\|\boldsymbol{d}(t)_{\text {uncontrolled }}\right\|}
$$

where $\|\cdot\|$ denotes the $l_{2}$-norm function. For quantification of acceleration, the cost functions are

$$
J_{3}=\frac{\max \left(\left|\ddot{x}(t)_{\text {controlled }}\right|\right)}{\max \left(\left|\ddot{x}(t)_{\text {uncontrolled }}\right|\right)}
$$

and

$$
J_{4}=\frac{\left\|\ddot{x}(t)_{\text {controlled }}\right\|}{\left\|\ddot{x}(t)_{\text {uncontrolled }}\right\|}
$$

Each cost function is an $n$-dimensional vector, thus providing quantification for each floor in the structure. Additionally, the control force demand is also quantified through a cost function provided in Ohtori et al., which is subsequently defined as 


$$
\boldsymbol{J}_{\mathbf{5}}=\frac{\max (|\boldsymbol{F}(t)|)}{W_{s}}
$$

where $\boldsymbol{F}(t)$ is the time history of the control force for each floor and $W_{s}$ is the seismic weight of the building based on the above ground mass of the structure.

As communication overhead is a common challenge associated with WSNs, and in particular when applied to control applications, an additional cost function,

$$
J_{6}=\frac{N P_{B I O}}{N P_{F S}},
$$

is introduced that compares the amount of data that is transmitted during the execution of the bio-inspired control algorithm versus the amount of data that is transmitted during the execution of the full-state LQR algorithm. In this cost index $N P_{B I O}$ is the number of peaks that are detected on all neuron boards across all receptor nodes, or all floors. $N P_{F S}$ is the number of data points obtained via traditional analog-todigital converter-based data acquisition at Nyquist rates combined across all floors.

\subsection{Weighting Matrices}

The weighting values for the bio-inspired control theory are derived using the three different methods described in Section 3 (BIO-LQR, ANN, and PSO). These weighting values are applied to the peak values that are transmitted by the cochlea-inspired sensing node and received at the motor neuron node. It is assumed that all of these weighting matrices are developed prior to execution of the control algorithm and no unsupervised training occurs, thus alleviating computational requirements at the motor neuron node and not affecting the overall control effectiveness.

The architecture of the weighting matrices is the same for BIO-LQR and PSO, resulting in a 55 x 5 matrix in each case. 55 represents the total number of "neurons" across all five floors (= 11 "neurons" per floor $\mathrm{x} 5$ floors) and 5 is the number of actuating nodes in the network. The weighting values for the BIO-LQR are developed using the output only LQR algorithm/with the state space model described in Equations 6 and 7. In the algorithm, $\boldsymbol{Q}$ and $\boldsymbol{R}$ are chosen using the commonly accepted Bryson's Rule [57], that establishes these values as proportional to the inverse of the square of the maximum acceptable displacement and control force, respectively. As such, $\boldsymbol{Q}$ is set to $10^{10} \times \widehat{\boldsymbol{C}}^{\boldsymbol{T}} \widehat{\boldsymbol{C}}$, and $\boldsymbol{R}$ is set to be $10^{-5.4} \times \boldsymbol{I}$, where $\boldsymbol{I}$ is the identity matrix.

In order to determine the weighting matrix for the PSO algorithm, an appropriate objective function is needed. In this case, the algorithm seeks to minimize the cost functions defined in Equations 16-19 by averaging the cumulative sum of each cost function overall all floors,

$$
f\left(x_{i j}\right)=\left(\sum_{m=1}^{4}\left(\sum_{l=1}^{n} J_{m, l}\right)\right) / 4
$$

where $n$ is the number of floors and equals 5 for the benchmark structure. In this objective function, each cost function is equally weighted so as to minimize both inter-story drift and acceleration. Each particle position, $x_{i j}$, represents a potential weighting matrix solution for the bio-inspired control theory and the objective function is evaluated on each position using the controlled structure's response subject to the El Centro earthquake. The algorithm is executed using 50 particles, each initialized as a vector of random values. As the search space is infinitely large, this relatively large number of initial positions allowed the system to span an adequate search space while also converging during a reasonable number of iterations. In particular, the number of iterations was limited to 100 , during which time the solution plateaued to a single solution for several iterations, indicating that this was a localized optimal solution. It is possible that a better solution would have been found if the iterations continued, but this also increased the 
possibility of the solution becoming over-trained to a specific earthquake, in this case El Centro, making it less generalizable to other earthquakes.

The weighting matrix for the ANN method is slightly more complex than those used for BIO-LQR and PSO due to the multi-layer network. For this study, the ANN consists of 55 input nodes, a single layer of 28 hidden nodes, and 5 output nodes. The input to the ANN is the inter-story displacement of each floor of the structure when subject to the El Centro earthquake after passing through the cochlea-inspired sensing nodes, resulting in 55 individual displacement signals. The output of the ANN represents the control force that is applied by the actuators. The ANN is trained over the time history of the El Centro earthquake signal using a target value that assumes that the ideal actuator is capable of applying control forces that exactly counteract the forces applied by the earthquake. Each time step in the ideal control force time history is considered to be a training point for the ANN. Using a batch training approach, all training points are presented to the ANN prior to the weight values being updated across the network. This is considered to be one iteration of training and iterations continued until the change in the average error function defined in Equation 12 was less than 1E-12, indicating acceptable convergence of the solution.

As a comparison to the bio-inspired control algorithm, a traditional full-state feedback LQR algorithm (FS-LQR) is also considered which assumed that all states (i.e., displacement and velocity) of all floors are measurable or estimated using techniques such as the Kalman filter. Based on the limitations described in [21], [23] it was assumed that the control sampling frequency was limited to $40 \mathrm{~Hz}$, thus subjecting the control effort to experimental constraints. For this comparative case, the bio-inspired sensing node was not included so the dynamics of the system are described by Equations 3 and 4 . $Q$ is also chosen as $10^{10} \times \boldsymbol{C}^{T} \boldsymbol{C}$, and $\boldsymbol{R}$ is set to be $10^{-5.4} \times \boldsymbol{I}$, where $\boldsymbol{I}$ is the identity matrix.

\subsection{Earthquake Simulation Results}

The structural response when excited by seismic base excitations is approximated using average Newmark integration [55]. The structure is subject to three different earthquake ground acceleration records: 1940 El Centro (SE), 1995 Kobe (JMA NS), and 1994 Northridge (Sylmar NS) (Figure 6). Each earthquake record is normalized to obtain a maximum acceleration of $1.0 \mathrm{~m} / \mathrm{s}^{2}$. Example time histories are provided in Figure 7 of both the uncontrolled and controlled structure response in order to demonstrate the effectiveness of the four control methods (i.e., BIO-LQR, ANN, PSO, and centralized LQR) in mitigating the effect of the earthquake. In particular, the inter-story drift of the fifth floor of the structure is shown for these scenarios as this floor is typically subject to large displacements resulting from the dominance of the first mode shape. In observing these time histories, however, it is evident that two of the methods, BIO-LQR and ANN, are not as effective at mitigating the response and these two methods are consequently removed in corresponding plots that are windowed over periods of large displacements in the uncontrolled system (Figures 7b, 7d, and 7f). In considering these sub-sections of the time history, it is evident that both the FS-LQR and PSO are able to effectively mitigate the effects of the earthquake on the structure, especially when considering the El Centro and Kobe earthquakes. For the Northridge earthquake, however, the PSO method is not as effective and is specifically unable to reduce the initial drift on this floor.

For each of the earthquakes, the control effectiveness of BIO-LQR, ANN, PSO, and centralized LQR is also considered using the displacement and acceleration cost functions provided in Equations 16-19 (Figure 8). To further understand the cumulative effect of these four different techniques, the sum of the cost functions associated with controlled displacement response (i.e., $\boldsymbol{J}_{1}$ and $\boldsymbol{J}_{2}$ ) for all floors is compared to the sum of the same cost functions using the uncontrolled responses for each earthquake. As the cost function is 1.0 if no control is applied, this results in the denominator being 10 ( $=5$ floors $\mathrm{x} 2$ cost functions), yielding the equation 

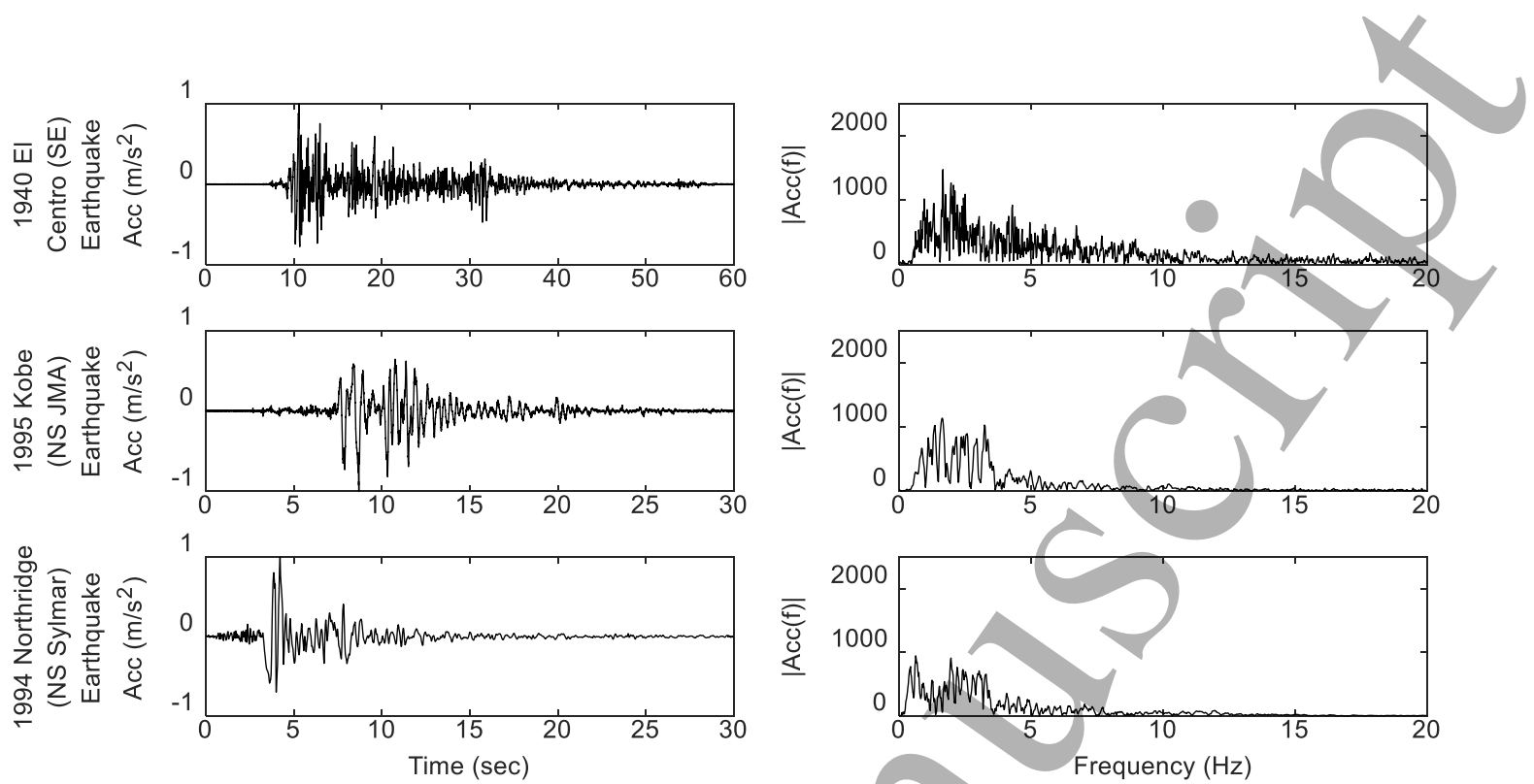

(a)

(b)

Figure 6. Seismic signals used as base excitation in simulation in time (a) and frequency (b) domains. SE: Southeast, NS:

Northsouth, JMA: Japan Meteorological Agency.

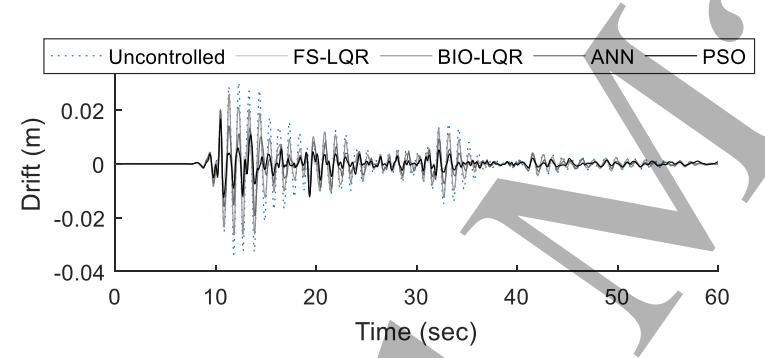

(a)

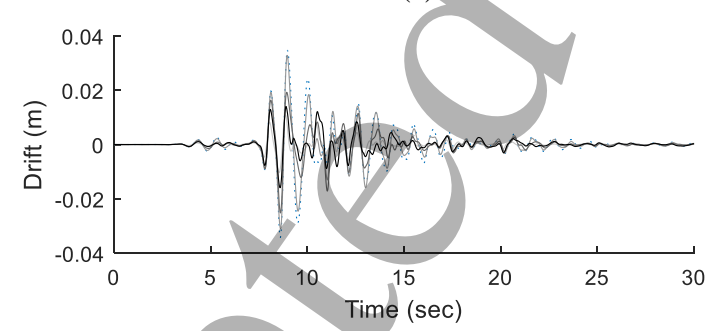

(c)

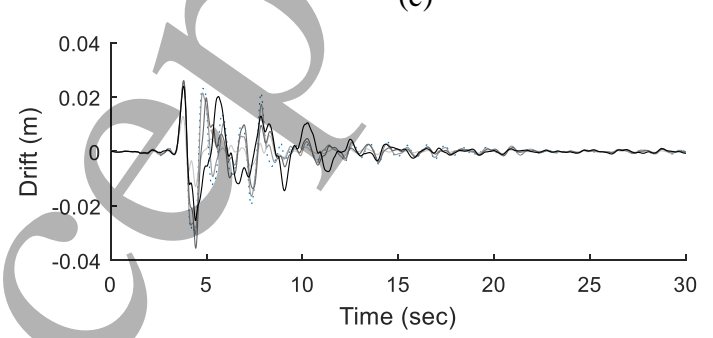

(e)

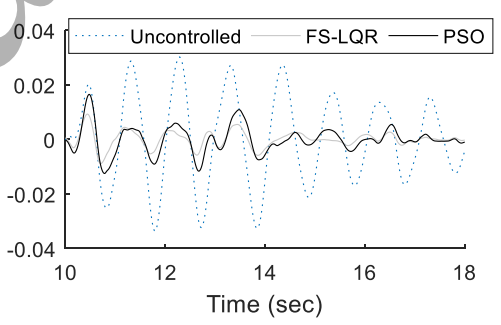

(b)

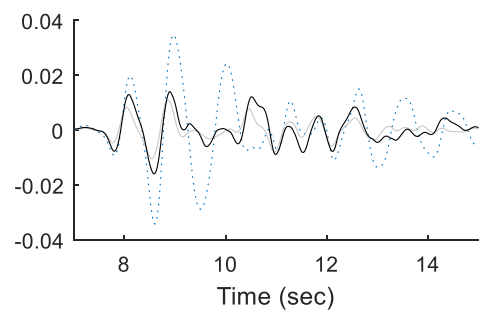

(d)

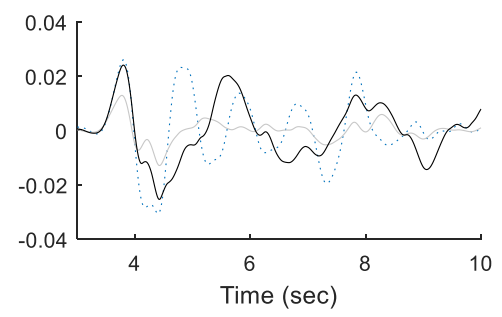

(f)

Figure 7. Inter-story drift response of the fifth floor when subject to the El Centro earthquake, shown on a full-time scale (a) and a sub-section of time (b), the Kobe earthquake, shown on a full time-scale (c) and a sub-section of time (d), and the Northridge earthquake, shown on a full time-scale (e) and a sub-section of time (f). FS-LQR: Full-State Linear Quadratic Regulator, BIO-LQR: Bio-inspired Linear Quadratic Regulator, ANN: Artificial Neural Network, PSO: Particle Swarm Optimization 

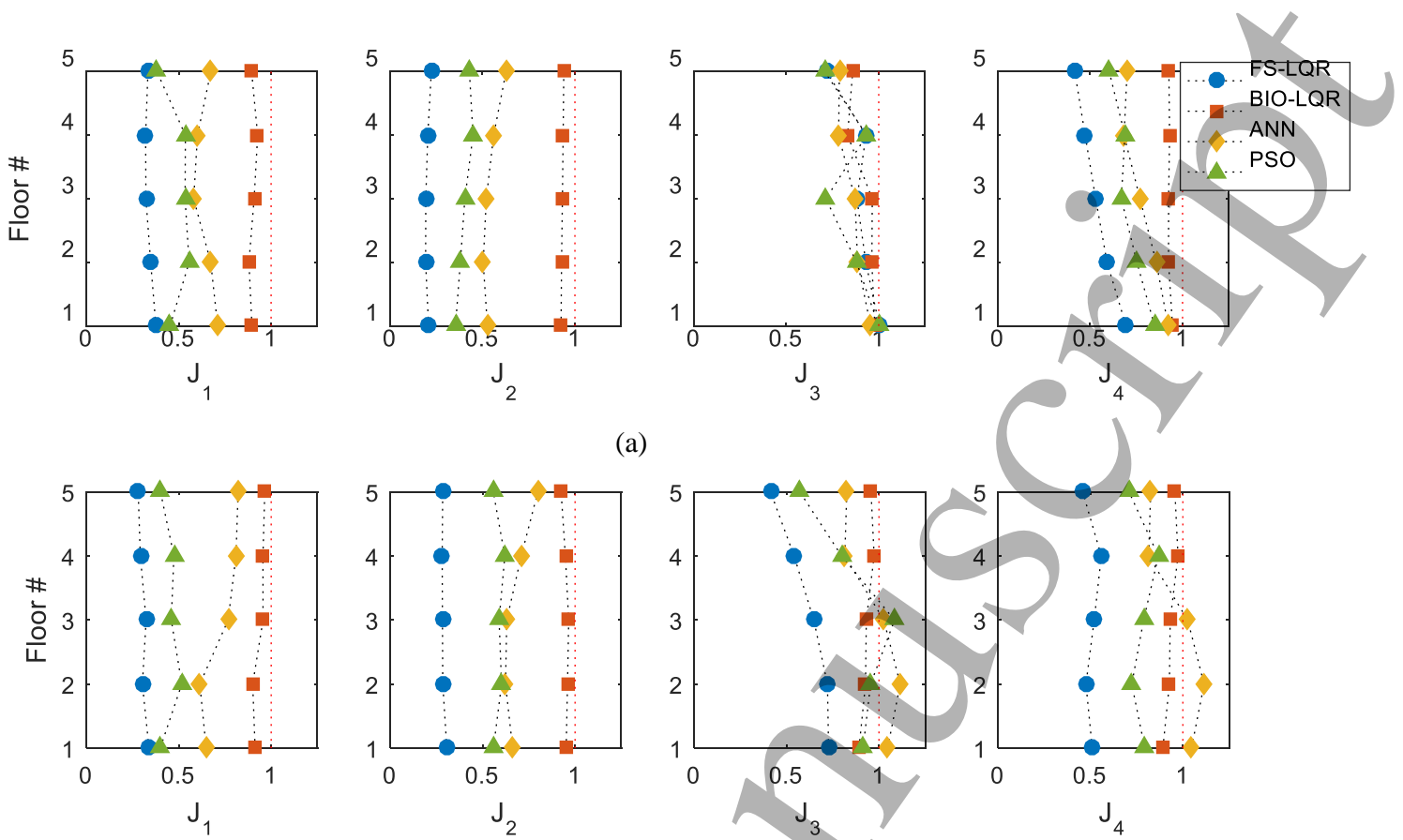

(a)
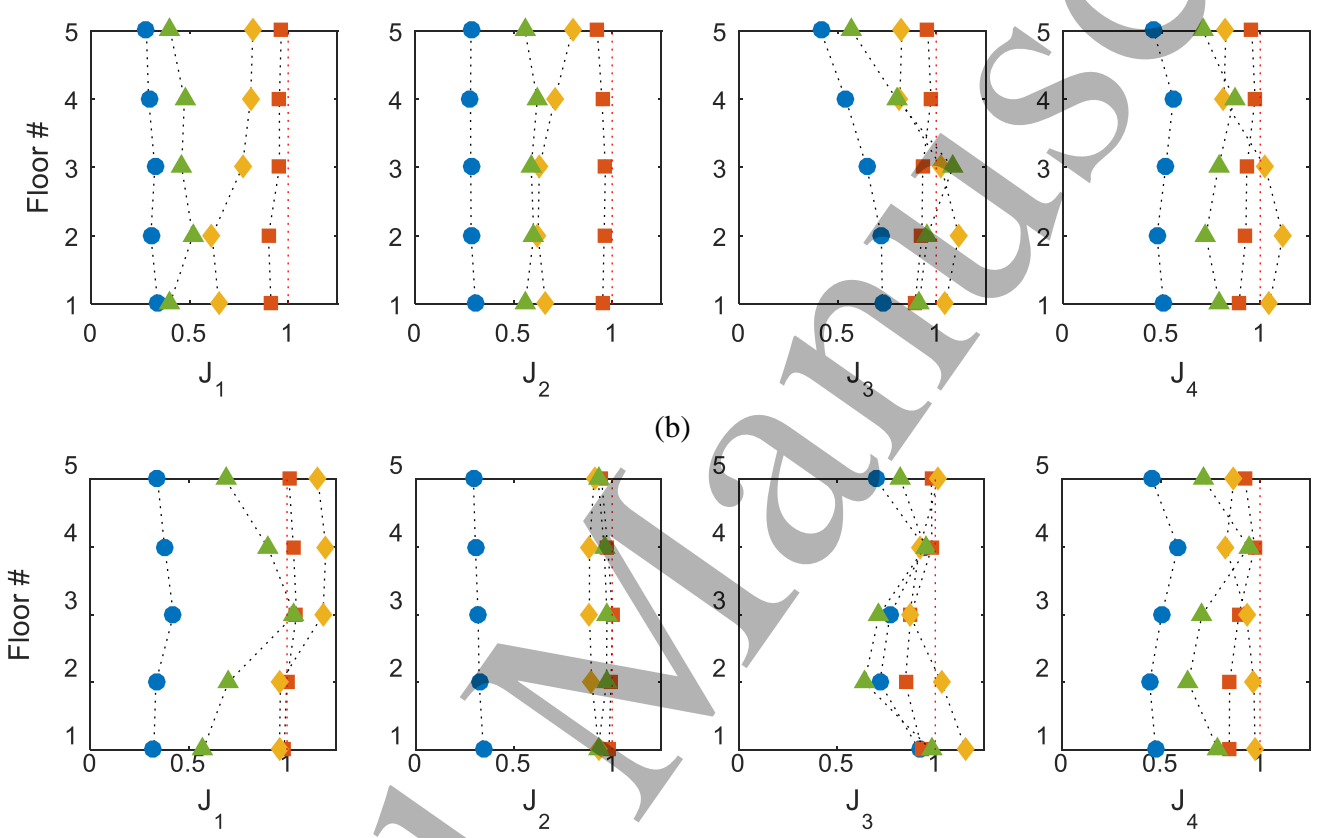

(c)

Figure 8. Cost functions for benchmark structure subject to El Centro earthquake (a), Kobe earthquake (b), and Northridge earthquake (c). FS-LQR: Full-State Linear Quadratic Regulator, BIO-LQR: Bio-inspired Linear Quadratic Regulator, ANN: Artificial Neural Network, PSO: Particle Swarm Optimization

$$
\operatorname{cost}=\left(\sum_{l=1}^{n} J_{1, l}+J_{2, l}\right) / 10
$$

and the results shown in Table 2. A similar relationship can be constructed for the cost functions associated with acceleration response (i.e., $\boldsymbol{J}_{3}$ and $\boldsymbol{J}_{4}$ ). From this analysis, it is evident that the centralized LQR method results in the most effective control, when considering both displacement and acceleration. The PSO method is the most effective method of the bio-inspired techniques, with both the ANN and the BIO-LQR methods not able to significantly reduce the structure's response. The ANN is more effective than the BIO-LQR in reducing the inter-story drift but on average it only minimally reduces acceleration and in some cases it will increase it. All three methods exhibit better control effectiveness for inter-story displacement than acceleration. 
Table 2. Ratios of combined cost functions, $\boldsymbol{J}_{1}+\boldsymbol{J}_{2}$ and $\boldsymbol{J}_{3}+\boldsymbol{J}_{4}$, averaged across floors for each bio-inspired control method, shown for all earthquakes

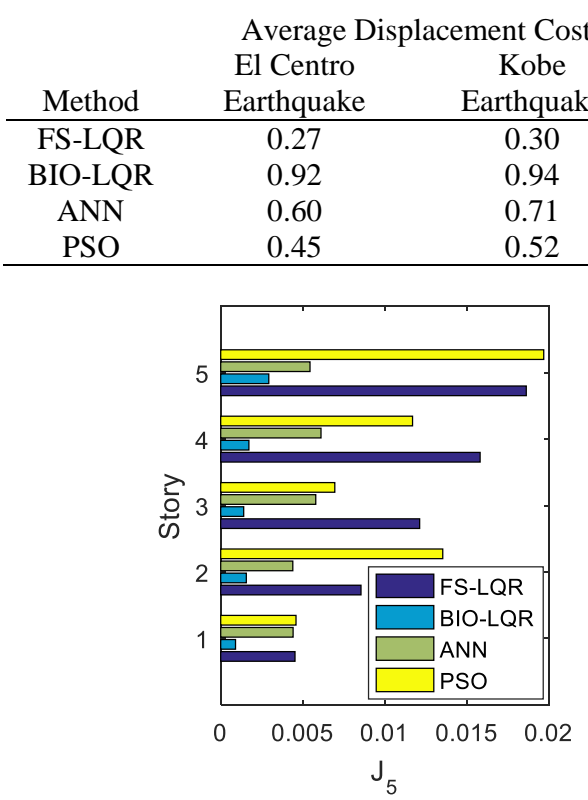

(a)

\begin{tabular}{|c|c|c|c|}
\hline Method & Earthquake & & Earthquak \\
\hline FS-LQR & 0.27 & & 0.30 \\
\hline BIO-LQR & 0.92 & & 0.94 \\
\hline ANN & 0.60 & & 0.71 \\
\hline PSO & 0.45 & & 0.52 \\
\hline 5 & ב & & \\
\hline 4 & 灵 & & \\
\hline $\mathbb{2}_{3}$ & 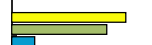 & & \\
\hline 2 & $\square$ & & \\
\hline & & & BIO-LQR \\
\hline 1 & 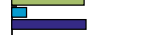 & & ANN \\
\hline & & & PSO \\
\hline & 0.005 & $\begin{array}{c}0.01 \\
\mathrm{~J}_{5}\end{array}$ & $0.015 \quad 0.02$ \\
\hline
\end{tabular}

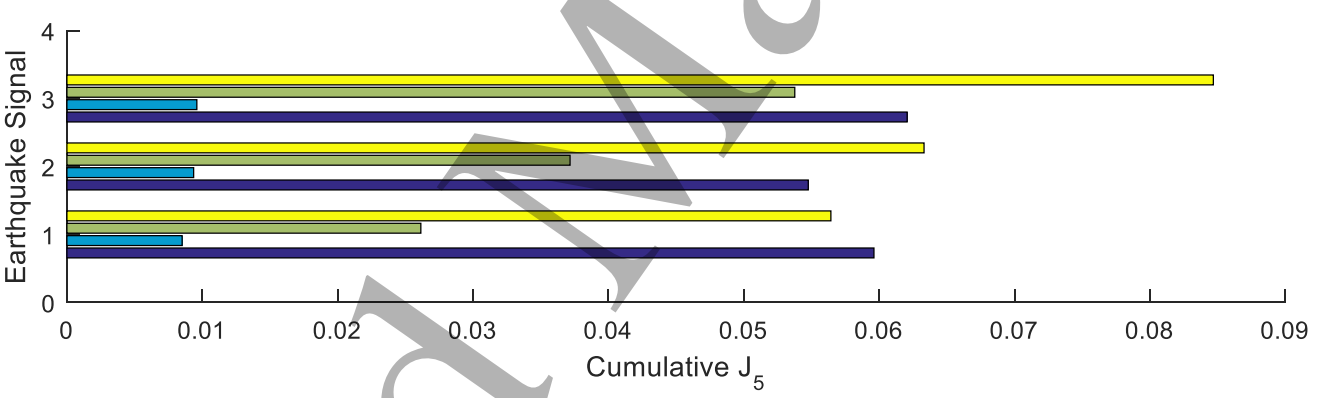

(d) unctions, $\boldsymbol{J}_{1}+\boldsymbol{J}_{2}$

Average Acceleration Cost Functions, $\boldsymbol{J}_{3}+\boldsymbol{J}_{4}$ El Centro Kobe Northridge $\begin{array}{cccc}\text { Earthquake } & \text { Earthquake } & \text { Earthquake } & \text { Earthquake } \\ 0.34 & 0.72 & 0.56 & 0.65\end{array}$

$\begin{array}{llll}0.34 & 0.72 & 0.56 & 0.65 \\ 0.99 & 0.92 & 0.94 & 0.91 \\ 0.99 & 0.82 & 0.97 & 0.96 \\ 0.87 & 0.78 & 0.82 & 0.79\end{array}$

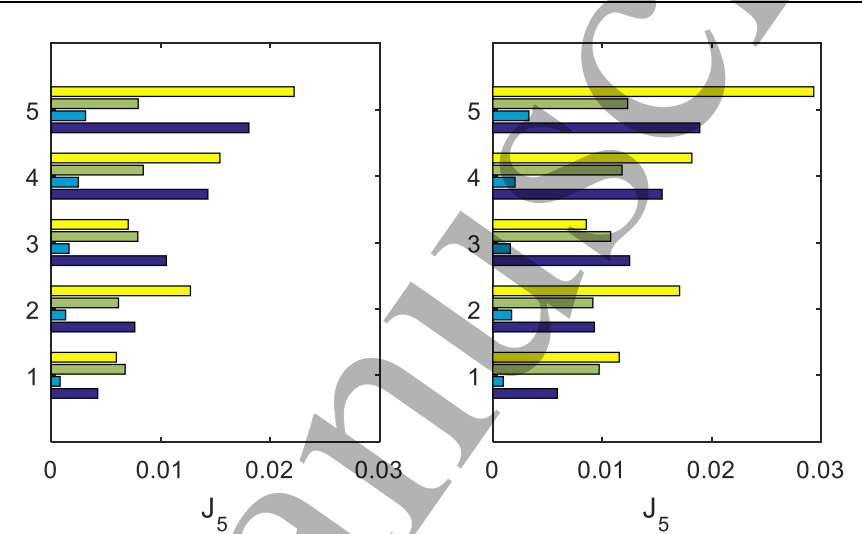

(b)

(c)

Figure 9. Control effort cost function for benchmark structure subject to El Centro earthquake (a), Kobe earthquake (b), and Northridge earthquake (c), and combined across all floors for each earthquake $(1=$ El Centro, $2=$ Kobe, $3=$ Northridge $)(d)$. FS-LQR: Full-State Linear Quadratic Regulator, BIO-LQR: Bio-inspired Linear Quadratic Regulator, ANN: Artificial Neural Network, PSO: Particle Swarm Optimization

The two other cost functions, $\boldsymbol{J}_{5}$ and $\boldsymbol{J}_{6}$, are also considered in order to fully quantify the control effectiveness of the four methods. The scaled actuator response values, or $\boldsymbol{J}_{5}$, are provided in Figure $9 \mathrm{a}$ through $9 \mathrm{c}$ for each earthquake. The cumulative actuator response summed across all floors is also shown in Figure 9d. The PSO places the greatest demand on the actuators, with a particularly large output from the actuator on the fifth floor. During training, the objective function for the PSO places no limitation on the actuator output and therefore it is expected that the optimal solution would place a large demand on this. The centralized LQR method has the second largest demand and does exceed the cumulative control force of the PSO for the Northridge earthquake. As expected, this large demand in both the centralized LQR and PSO translate into more effective control, as depicted by the reduced control effectiveness cost functions $\left(\boldsymbol{J}_{1}\right.$ through $\left.\boldsymbol{J}_{4}\right)$, Both the BIO-LQR and the ANN methods require significantly less actuator output but they also produce less effective control than the other two methods. The weights for the ANN method are produced by assuming an idealized actuator output that perfectly counterbalances the earthquake force at each floor but when compared to the trends demonstrated by the centralized LQR and PSO, it could be concluded that a larger actuator output from this method may 
Table 3. Ratios of communication cost function, $\boldsymbol{J}_{6}$, for each bio-inspired control method and all earthquakes

\begin{tabular}{cccc} 
Method & $\begin{array}{c}\text { El Centro } \\
\text { Earthquake }\end{array}$ & Kobe Earthquake & $\begin{array}{c}\text { Northridge } \\
\text { Earthquake }\end{array}$ \\
\hline FS-LQR & 1.00 & 1.00 & 1.00 \\
BIO-LQR & 1.22 & 0.64 & 0.49 \\
ANN & 1.21 & 0.70 & 0.49 \\
PSO & 1.28 & 0.89 & 0.63 \\
\hline
\end{tabular}

Table 4. Ratios of combined cost functions, $\boldsymbol{J}_{1}+\boldsymbol{J}_{2}$ and $\boldsymbol{J}_{3}+\boldsymbol{J}_{4}$, averaged across floors and earthquakes and cumulative control force, $\boldsymbol{J}_{5}$, for ANN and PSO algorithms using just one training earthquake or three training earthquakes

\begin{tabular}{ccccc} 
Method & $\begin{array}{c}\text { Average } \\
\text { displacement cost } \\
\text { functions }\left(\boldsymbol{J}_{1}, \boldsymbol{J}_{2}\right)\end{array}$ & $\begin{array}{c}\text { Average } \\
\text { acceleration cost } \\
\text { functions }\left(\boldsymbol{J}_{3}, \boldsymbol{J}_{4}\right)\end{array}$ & $\begin{array}{c}\text { Average combined } \\
\text { cost functions }\end{array}$ & $\begin{array}{c}\text { Cumulative control } \\
\text { force cost function, } \boldsymbol{J}_{5}\end{array}$ \\
\hline ANN - El Centro Only & 0.73 & 0.87 & 0.80 & 0.21 \\
ANN - 3 Earthquakes & 0.73 & 0.86 & 0.80 & 0.21 \\
PSO - El Centro Only & 0.58 & 0.77 & 0.68 & 0.37 \\
PSO - 3 Earthquakes & 0.54 & 0.78 & 0.66 & 0.36 \\
\hline
\end{tabular}

produce more effective control. A scaling term was introduced into the weights between the hidden layer and output layer in an attempt to increase the actuator output and thereby improve the control effectiveness, but this produced an unstable result. Additionally, the BIO-LQR weighting matrix is constrained by the $\boldsymbol{Q}$ and $\boldsymbol{R}$ matrices and as such, different values could improve the control effectiveness. Numerous combinations were explored and the general resulting trend was that as the displacement cost functions decreased the acceleration cost functions increased.

The communication constraints are considered using $\boldsymbol{J}_{6}$, which compares the number of packets transmitted via the bio-inspired technique to the number of packets transmitted using traditional Nyquist sampling techniques. Table 3 shows this cost function for the four methods across the three different earthquakes. The bio-inspired techniques transmit less data for all earthquakes except for the El Centro earthquake. This earthquake, as compared to the other two earthquakes, spans a much broader frequency band which causes the cochlea-inspired sensing node to transmit more peaks [40]. These communication savings across the sensing and actuating network, in general, results in power savings of the overall system, which helps to justify the lower control performance in these methods. To further improve the power savings of the bio-inspired control method, especially when consider relatively broad band earthquake signals such as the El Centro, pruning techniques could be applied to remove "neuron" boards that do not significantly impact the control effectiveness. This will be explored in future work.

One final consideration is that the PSO and ANN were both trained using only the El Centro earthquake and this could lead to questions about the generalizability of these two methods. To address this, these two methods were trained using the El Centro, Kobe, and Northridge earthquakes and then were also evaluated for control effectiveness using these three earthquakes, as well as the 2010 Chile (ANGOL) earthquake and 1989 Loma Prieta (CORRALITOS) earthquakes. Training the ANN on the three earthquakes has little effect on the weighting values and as a result, had no effect on the control effectiveness or actuator demands. By training the PSO algorithm on three earthquakes, however, the solutions became slightly more general, resulting in minor improvements in the cost function across all five earthquakes (Table 4). Additionally, the average maximum actuator output is slightly reduced as well, indicating that this increased generalizability is an improvement. 


\subsection{Conclusions}

While feedback control systems integrated into civil infrastructure is not a new area of research, several challenges of the technology, such as computational delays and communication constraints, have prevented their widespread adoption. This study proposed a bio-inspired control algorithm that leverages front-end signal processing to enable streamlined control at the actuating node, thus overcoming many of these challenges. The control algorithm is reduced down to a simplistic weighted combination of the inputs, similar to mechanisms employed by the central nervous system. The weights of this algorithm were developed using three different techniques: optimal control theory, artificial neural networks, and particle swarm optimization. The effectiveness of these three different methods was assessed in simulation on a five story benchmark structure. While the traditional full-state optimal control theory does outperform the bio-inspired control algorithm, the PSO technique in particular offers a competitive alternative in overall control effectiveness. The drawback of the PSO technique, however, it is that it does place a higher demand on the actuators, as compared to the other techniques and this must be considered when completing an experimental validation of the techniques. Additionally, the bio-inspired control techniques do offer power savings across the entire network as they typically transmit less data.

Future work will include experimental validation of the bio-inspired algorithm using the BIO-LQR, ANN, and PSO techniques, as well as the full-state feedback control system. This experimental validation will use WSNs for communicating information about the structural response, as well as commanding the actuator. To implement the full-state feedback method, a Kalman filter will be implemented which will inhibit the real-time capabilities of the full-state feedback method, due to communication sequencing and computational delays. The other three methods, however, will leverage the real-time front-end signal processing capabilities of the cochlea-inspired sensing node which will alleviate computations at the actuating node and prevent delays in the overall control system.

\subsection{Acknowledgements}

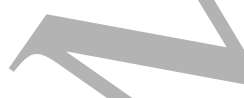

The authors gratefully acknowledge the generous support offered by the National Science Foundation (NSF) through grant CMMI-1662655, as well as the Howard Hughes Medical Institute (HHMI) Undergraduate Science Education Program. The authors would also like to thank Anne O'Donnell (Hope College) who assisted with the research effort.

\section{References}

[1] J. T. P. Yao, “Concept of Structural Control,” J. Struct. Div., vol. 98, no. 7, pp. 1567-1574, 1972. [2] G. W. Housner et al., "Structural control: past, present, and future," J. Eng. Mech., vol. 123, no. 9, pp. 897-971, 1997.

[3] B. F. Spencer and S. Nagarajaiah, "State of art structural control," J. Struct. Eng., vol. 129, no. 7, pp. 845-856, 2003.

[4] T. Soong, Active structural control: Theory and practice. Essex, U.K.: Longman Scientific and Technical, 1990.

[5] M. D. Symans and M. C. Constantinou, "Semi-active control systems for seismic protection of structures: a state-of-the-art review,” Eng. Struct., vol. 21, no. 6, pp. 469-487, 1999.

[6] N. Kurata, T. Kobori, M. Takahashi, N. Niwa, and H. Midorikawa, "Actual seismic response controlled building with semi-active damper system," Earthq. Eng. Struct. Dyn., vol. 28, no. 11, pp. 1427-1447, 1999.

[7] S. N. Pakzad, G. L. Fenves, S. Kim, and D. E. Culler, "Design and implementation of scalable wireless sensor network for structural monitoring," J. Infrastruct. Syst., vol. 14, no. 1, pp. 89-101, 2008. [8] S. Jang et al., "Structural health monitoring of a cable-stayed bridge using smart sensor technology: deployment and evaluation," Smart Struct. Syst., vol. 5, no. 6, pp. 439-459, 2010.

[9] M. Kurata et al., "Internet-enabled wireless structural monitoring systems: development and 
permanent deployment at the New Carquinez suspension bridge,” J. Struct. Eng., vol. 139, no. 10, pp. 1688-1702, 2013.

[10] F. Moreu, R. E. Kim, and B. F. Spencer, "Railroad bridge monitoring using wireless smart sensors," Struct. Control Health Monit., 2016.

[11] M. Ceriotti et al., "Monitoring heritage buildings with wireless sensor networks: the Torre Aquila deployment," in International Conference on Information Processing in Sensor Networks, 2009, pp. 277 288.

[12] N. Kurata, B. F. Spencer, M. Ruiz-Sandoval, Y. Miyamoto, and Y. Sako, "A study on building risk monitoring using wireless sensor network MICA-Mote," in 1st International Conference on Structural Health Monitoring and Intelligent Infrastructure, 2003.

[13] J. Kim and J. P. Lynch, "Autonomous decentralized system identification by Markov parameter estimation using distributed smart wireless sensor networks," J. Eng. Mech., vol. 138, no. 5, pp. 478-490, 2012.

[14] R. Rolfes, S. Zerbst, G. Haake, J. Reetz, and J. P. Lynch, "Integral SHM-System for Offshore Wind Turbines Using Smart Wireless Sensors," in 6th International Workshop on Structural Health Monitoring, 2007.

[15] R. A. Swartz, J. P. Lynch, S. Zerbst, B. Sweetman, and R. Rolfes, "Structural monitoring of wind turbines using wireless sensor networks," Smart Struct. Syst., vol. 6, no. 3, pp. 183-196, 2010.

[16] B.-G. Paik, S.-R. Cho, B.-J. Park, D. Lee, B.-D. Bae, and J.-H. Y un, "Characteristics of wireless sensor network for full-scale ship application," J. Mar. Sci. Technol., vol. 14, no. 1, pp. 115-126, 2009.

[17] R. A. Swartz et al., "Hybrid wireless hull monitoring system for naval combat vessels," Struct. Infrastruct. Eng., vol. 8, no. 7, pp. 621-638, 2012.

[18] C.-H. Loh et al., "Experimental verification of a wireless sensing and control system for structural control using MR dampers," Earthq. Eng. Struct. Dyn., vol. 36, no. 10, pp. 1303-1328, 2007. [19] Y. Wang, A. Swartz, J. P. Lynch, K. H. Law, K.-C. Lu, and C.-H. Loh, "Wireless feedback structural control with embedded computing," in SPIE-Health Monitoring and Smart Nondestructive Evaluation of Structural and Biological Systems, 2006.

[20] J. P. Lynch, Y. Wang, R. A. Swartz, K.-C. Lu, and C.-H. Loh, "Implementation of a closed-loop structural control system using wireless sensor networks," Struct. Control Health Monit., vol. 15, pp. 518-539, 2008.

[21] B. F. Spencer et al., "Recent advances in wireless smart sensors for multi-scale monitoring and control of civil infrastructure," J. Struct. Health Monit., vol. 6, pp. 17-41, 2016.

[22] Y. Wang, R. A. Swartz, J. P. Lynch, K. H. Law, and C.-H. Loh, "Performance evaluation of decentralized wireless sensing and control in civil structures," in Proceedings of SPIE, Vol. 6531, 2007. [23] R. A. Swartz and J. P. Lynch, "Strategic network utilization in a wireless structural control system for seismically excited structures," J. Struct. Eng., vol. 135, no. 5, pp. 597-608, 2009.

[24] R. Verdoljak and L. E. Linderman, "Sparse feedback structures for control of civil systems," Struct. Control Health Monit., vol. 23, pp. 1334-1349, 2016.

[25] Y. Wang, J.P. Lynch, and K. H. Law, "Decentralized H-infinity controller design for large-scale civil structures,” Earthq. Eng. Struct. Dyn., vol. 38, pp. 377-401, 2009.

[26] Y. Wang and K. H. Law, "Structural control of multi-subnet wireless sensing feedback: experimental validation of time-delayed decentralized h-infinity control design," Adv. Struct. Eng., vol. 14, no. 1 , pp. $25-39,2011$.

[27] J. G. Nicholls, A. R. Martin, B. G. Wallace, and P. A. Fuchs, From Neuron to Brain, 4th ed. Sunderland, MA: Sinauer Associate, 2001.

[28] A. V. M. Herz, T. Gollisch, C. K. Machens, and D. Jaeger, "Modeling single-neuron dynamics and computations: a balance of detail and abstraction," Science, vol. 314, pp. 80-85, 2006.

[29] G. E. Loeb and C. Ghez, "The motor unit and muscle action," in Principles of Neural Science, 4th ed., E. Kandel, J. Schwartz, and T. Jessell, Eds. New York: McGraw-Hill, 2000.

[30] E. P. Gardner and J. H. Martin, "Coding of Sensory Information," in Principles of Neural Science, 4th ed., E. R. Kandel, J. H. Schwartz, and T. M. Jessell, Eds. New York: McGraw-Hill, 2000, pp. 
$411-429$.

[31] W. Bialek, F. Rieke, R. R. de Ruyter Van Steveninch, and D. Warland, "Reading a neural code," Science, vol. 252, pp. 1854-1857, 1991.

[32] E. R. Kandel, "Nerve cells and behavior," in Principles of Neural Science, 4th ed., E. R. Kandel, J. H. Schwartz, and T. M. Jessell, Eds. New York: McGraw-Hill, 2000, pp. 19-35.

[33] W. B. J. Kristan, R. L. Calabrese, and W. O. Friesen, "Neuronal control of leech behavior," Prog. Neurobiol., vol. 76, pp. 279-327, 2005.

[34] J. M. Gray, J. J. Hill, and C. I. Bargmann, "A circuit for navigation in Caenorhabditis elegans," PNAS, vol. 102, no. 9, pp. 3184-3191, 2005.

[35] A. Marin-Burgin, W. B. J. Kristan, and K. A. French, "From synapse to behavior: development of a sensory-motor circuit in the leech," Dev. Neurobiol., vol. 68, no. 6, pp. 779-787, 2008.

[36] H. S. Milner-Brown, R. B. Stein, and R. Yemm, "Changes in firing rate of human motor units during linearly changing voluntary contractions," J. Physiol., vol. 230, pp. 371-390, 1973.

[37] E. Henneman, G. Somjen, and D. O. Carpenter, "Functional significance of cell size in spinal motoneurons," J. Neurophysiol., vol. 28, no. 3, pp. 560-580, 1965.

[38] T. C. Cope and M. J. Pinter, "The size principle: still working after all these years," Physiology, vol. 10, no. 6, pp. 280-286, 1995.

[39] D. M. Wolpert and Z. Ghahramani, "Computational principles of movement neuroscience," Nat. Neurosci., vol. 3, pp. 1212-1217, 2000.

[40] C. A. Peckens, J. P. Lynch, and G. Heo, "Resource efficient wireless sensor network architecture based on bio-mimicry of the mammalian auditory system," J. Intell, Mater. Syst. Struct., vol. 26, no. 1, pp. 79-100, 2015.

[41] P. Dallos, "Overview: cochlear neurobiology," in The cochlea, P. Dallos, A. N. Popper, and R. R. Fay, Eds. New York, NY, 1996, pp. 1-43.

[42] C. A. Peckens and J. P. Lynch, "Utilizing the cochlea as a bio-inspired compressive sensing technique," Smart Mater. Struct., vol. 20, no. 105027, 2013.

[43] T. V. Bliss and T. Lomo, "Long-lasting potentiation of synaptic transmission in the dentate area of the anaesthetized rabbit following stimulation of hte perforant path," J. Physiol., vol. 232, no. 2, pp. 331-356, 1973.

[44] D. O. Hebb, The organizaiton of behavior: A neuropsychological theory. New York: Wiley, 1949.

[45] R. E. Kalman, "Contributions to the theory of optimal control," Boletin Soc. Mat. Mex. Repr., vol. 5, pp. 102-119, 1960.

[46] G. Welch and G. Bishop, "An Introduction to the Kalman Filter," Chapel Hill, NC, 2006.

[47] F. L. Lewis and V. L. Syrmos, Optimal Control. New York: John Wiley and Sons, 1995.

[48] K. Hornik, M. Stinchcombe, and H. White, "Multilayer feedforward neural networks are universal approximators," Neural Netw., vol. 2, no. 5, pp. 359-366, 1989.

[49] G. Cybenko, "Approximation by Superpositions of a Sigmoidal Function," Math. Control Signals Syst., vol. 2, pp. 303-314, 1989.

[50] R. O. Duda, P. E. Hart, and D. G. Stork, "Multilayer Neural Networks," in Pattern Classification, 2nd ed., New York, NY: Wiley-Interscience, 2001.

[51] J. Kennedy and R. Eberhart, "Particle Swarm Optimization," in IEEE International Conference on Neural Networks, 1995.

[52] Y.Shi and R. Eberhart, "A Modified particle swarm optimizer," in IEEE International Conference on Evolutionary Computation Proceedings, 1998.

[53] R. Poli, J. Kennedy, and T. Blackwell, "Particle Swarm Optimization: An Overview," Swarm Intell., vol. 1, no. 1, pp. 33-57, 2007.

[54] Y. Wang, "Wireless sensing and decentralized control for civil structures: theory and implementation," Stanford University, 2007.

[55] A. Chopra, Dynamics of Structures:Theory and Applications to Earthquake Engineering, 5th ed. Hoboken, NJ: Pearson, 2017. 
[56] Y. Ohtori, R. E. Christenson, B. F. Spencer, and S. J. Dyke, "Benchmark control problems for seismically excited nonlinear buildings," J. Eng. Mech., vol. 130, no. 4, 2004.

[57] G. F. Franklin, J. D. Powell, and A. Emami-Naeini, Feedback Control of Dynamic Systems, 6th ed. Saddle River, NJ: Prentice Hall, 2010. 